\title{
The bovine viral diarrhea virus (BVDV) NS3 protein, when expressed alone in mammalian cells, induces apoptosis which correlates with caspase-8 and caspase- 9 activation
}

\author{
Marie-Claude ST-LouIs ${ }^{\mathrm{a}}$, Bernard MASSIE ${ }^{\mathrm{b}, \mathrm{c}, \mathrm{d}}$, Denis ARCHAMBAUlT ${ }^{\mathrm{a} *}$ \\ a Department of Biological Sciences, University of Québec at Montréal, PO Box 8888, \\ Succursale Centre-Ville, Montréal, Québec, H3C 3P8, Canada \\ b Department of Molecular Biology, Biotechnology Research Institute, Montréal, Québec, Canada \\ ${ }^{c}$ INRS-IAF, University of Québec, Laval, Québec, Canada \\ ${ }^{\mathrm{d}}$ Department of Microbiology and Immunology, Faculty of Medicine, Montréal University, \\ Québec, Canada
}

(Received 22 June 2004; accepted 14 October 2004)

\begin{abstract}
The bovine viral diarrhea virus (BVDV) strains exist as two biotypes, cytopathic (cp) and noncytopathic (ncp), according to their effects on tissue culture cells. It has been previously reported that cell death associated to cp BVDV in vitro is mediated by apoptosis. Here, experiments were conducted to determine the involvement of the NS3 protein in the induction of apoptosis. The NS3- and NS3 $\triangle 50$ (deleted from the NH2-terminal 50 amino acids)-cDNA encoding sequences of BVDV NADL cp reference strain were cloned into adenoviral vectors (AdV) from which the BVDV gene of interest could be expressed from a tetracycline-responsive promoter. A549tTA cells infected in vitro with NS3 or NS3 $\Delta 50$-expressing AdV showed cytopathic changes characterized by cell rounding and detachment, and nucleus chromatin condensation. DNA fragmentation assays, cytochrome c release, and activation of cellular caspases performed on these infected cells clearly correlated with the observed cytopathic changes with apoptosis. The BVDV NS3 $\Delta 50$-induced apoptotic process was inhibited by caspase-8- and -9-specific peptide inhibitors (Z-IETD-FMK and Z-LEHD-FMK). Furthermore, apoptosis was inhibited in cells expressing the R1 subunit of herpes simplex virus type 2 ribonucleotide reductase (HSV2-R1) or hsp70, two proteins which are known to inhibit apoptosis associated with caspase- 8 activation and cytochrome c release-dependent caspase-9 activation, respectively. Given that HSV2-R1, a specific inhibitor of the caspase-8 activation pathway, efficiently suppressed apoptosis and also prevented caspase- 9 activation, the overall results indicate that the BVDV NS3/NS3 $\Delta 50$ induces apoptosis initiated by caspase- 8 activation and subsequent cytochrome $\mathrm{c}$ release-dependent caspase-9 activation.
\end{abstract}

bovine viral diarrhea virus / NS3 protein / apoptosis / caspases

\section{INTRODUCTION}

Apoptosis is considered as the physiological form of cell death that occurs during embryonic development, tissue remodeling and tumor regression [39]. Among stimuli that have been associated with cell apoptosis are infections with mammalian DNA

* Corresponding author: archambault.denis@uqam.ca 
and RNA viruses [13]. Viruses possess various biochemical and genetic mechanisms to evade and/or induce apoptosis in infected cells through interactions at different stages of the apoptotic pathway. The fact that virusderived proteins have individually been shown to inhibit or induce apoptosis is consistent with this fact [13].

Two major regulatory pathways of apoptosis have been identified. The extrinsic pathway is triggered by a receptor/ligand interaction mechanism which involves the recruitment of the proximal regulatory caspase-8 to the death receptor complex, resulting in cleavage of the effector caspases-3 and -7, whereas the intrinsic pathway is associated with the release of cytochrome c from mitochondria and the subsequent activation of Apaf-1, causing multimerization and autocleavage of caspase- 9 and the further cleavage of the effector caspases [29, 32, $37,41,45]$. A third pathway of apoptosis may exist, mediated by endoplasmic reticulum (ER) stress and characterized by cleavage of caspase-12 [31, 36], further indicating the complex nature of the regulatory pathways of apoptosis.

Bovine viral diarrhea virus (BVDV) is a world-wide distributed pathogen of cattle $[28,43]$. This virus, together with classical swine fever virus (CSFV) and border disease virus (BDV) in the ovine, belongs to the genus Pestivirus of the Flaviviridae family [43]. BVDV often causes subclinical infections or mild clinical signs in cattle. However, fetuses infected in the early stage of intrauterine development may become immunotolerant adults which are persistently infected with BVDV, and which will propagate the virus within the herd [28, 43]. Such animals will develop a severe fatal syndrome, termed mucosal disease (MD), characterized by profound lymphocytolysis and severe widespread ulcerations of the digestive tract [28, 43].

The pestiviral genome is a positive, singlestranded RNA molecule of usually $12.3 \mathrm{~kb}$ in length that encodes one polyprotein of about 4000 amino acids, which is co- and post-translationally processed by cell- and virus-derived proteases to give rise to the mature structural and non structural viral proteins [28]. The hallmark of BVDV strains is that they exist as two biotypes, cytopathic (cp) and noncytopathic (ncp), according to their effects on tissue culture cells [22]. NS3 is expressed only in cells infected with the cp strains [22, 27].

Cells infected with the cp biotype of BVDV have been shown to undergo apoptosis [46]. This apoptosis process has been associated with the accumulation of large amounts of viral RNA [44], cleavage of poly(ADP-ribose) polymerase (PARP) [14], mitochondrial-dependent caspase-9 activation [11], and endoplasmic reticulum (ER) stress induction that correlates with the activation of caspase-12 [18]. It is prevented by certain anti-oxidants [40]. In this report, we demonstrate that the NS3 protein of BVDV expressed from an inducible promoter in an adenovirus vector (AdV) induces programmed cell death (apoptosis) in vitro. Experiments conducted with an AdV expressing a deletion mutated form of the NS3 protein (NS3 $\Delta 50)$ have shown that the NH2-terminal fifty amino acids of the protein are dispensable for NS3-induced apoptosis. Finally, we showed that the BVDV NS3/NS3 $\Delta 50$-induced apoptotic process was associated with both caspase- 8 and cytochrome c release-dependent caspase- 9 activation.

\section{MATERIALS AND METHODS}

\subsection{Cells and viruses}

The cp NADL reference strain of BVDV (ATCC\# VR-534) was propagated in BVDVfree MDBK cells [4]. BMAdE1 220-8, 293A, and 293rtTA [15, 25, 26] were propagated in antibiotic-free Dulbecco minimal essential medium (DMEM) with high glucose concentration supplemented with $10 \%$ tetracycline-free fetal bovine serum (FBS) (Clontech Laboratories Inc., Palo Alto, 
Table I. Oligonucleotide primers used to generate recombinant plasmids containing BVDV NS3-and NS3 $\Delta 50$-encoding nucleic acid sequences.

\begin{tabular}{|c|c|c|}
\hline Recombinant plasmids & Nucleotide sequences $\left(5^{\prime} \rightarrow 3^{\prime}\right)$ & Sense \\
\hline \multirow[t]{3}{*}{ pBS/AdV (NS3) } & CAAAGTTTAAACGATCCACCATGGGA CAT CAC CAT CAC & + \\
\hline & CAT CAC GGGCCTGCCGTGTGTAAGAAG & \\
\hline & CAAAGTTTAAAC TCACAACCCGGTCACTTGCTTCAGT & - \\
\hline \multirow[t]{3}{*}{ pBS/Ad (NS3 $\triangle 50)$} & CAAAGTTTAAACGATCCACCATGGGA CAT CAC CAT CAC & \\
\hline & CAT CAC GGTCTGGAGACTGCCTGGGCTTA & + \\
\hline & CAAAGTTTAAAC TCACAACCCGGTCACTTGCTTCAGT & - \\
\hline
\end{tabular}

The underlined nucleotides (GTTTAAAC) refer to the restriction endonuclease cleavage site PmeI. The initiation (ATG), termination ( $\underline{(T C A)}$ ) and histidine codons (CAT and $C A C)$ are also shown.

USA). 293A and BMAdE1 cells were used for AdV generation and amplification [15, 26] whereas 293rtTA cells were used to assess the expression of the protein of interest and to titrate the adenovirus stocks by flow cytometry monitoring of GFP expression [26]. A549 cells (derived from human lung carcinoma tissues) genetically transformed to express the tetracycline transactivation factor (tTA) (A549tTA) [25] were used in cell apoptosis experiments conducted with the AdV. African Green Monkey kidney (Vero), canine fetal thymus (Cf2Th) and Mardin-Darby bovine kidney (MDBK) continuous cell lines were also tested to determine whether the pestiviral protein also induces apoptosis in these cells.

\subsection{Viral RNA isolation and oligonucleotide primers}

Viral genomic RNA was extracted using the guanidium isothiocyanate method from the supernatant of infected MDBK cells as described [1]. The oligonucleotide primers for PCR amplification of nucleic acid sequences that encode BVDV NS3 protein (nucleotides 5423 to 7471 of the viral genome; amino acids 1 to 683 of NS3) and a truncated form of NS3 (NS3 $\Delta 50$ ) (nucleotides 5573 to 7471 of the viral genome; amino acids 51 to 683 of NS3) were selected according to the BVDV NADL strain genomic sequence (Genbank Database accession number M31182), and to the predicted $\mathrm{NH} 2-$ and $\mathrm{COOH}$-termini of the protein [48]. Primers (listed in Tab. I) contained short 5' extensions in which restriction endonuclease cleavage sites, and initiation or termination codons were present for cloning/subcloning purposes. The decision to clone the sequences encoding the whole and truncated form of the NS3 protein into the eukaryotic adenovirus expression system was taken from the beginning because a negligible expression level of NS3 in bacterial cells from a prokaryotic expression construct was achieved. Deleting the NH2terminal of NS3, which was predicted to contain several hydrophobic amino acid residues [23], resulted in a high expression level of the protein. Thus, on the basis of these results, recombinant adenoviruses carrying the NS3 and NS3 350 -encoding sequences were constructed to maximize the likelihood of expressing the pestiviral protein in mammalian cells.

\subsection{Reverse transcription-PCR amplification, cloning, and sequencing}

BVDV genomic RNA was converted to complementary DNA (cDNA) by reverse transcription using random hexadeoxyribonucleotides $(\mathrm{pd}(\mathrm{N}) 6$; Pharmacia Biotech Inc., Uppsala, Sweden) as previously described 
[42]. The cDNA was then amplified using the appropriate primer pair by 35 successive cycles of denaturation at $95^{\circ} \mathrm{C}$ for $1.5 \mathrm{~min}$, primer annealing at $48{ }^{\circ} \mathrm{C}$ for $1.5 \mathrm{~min}$, and DNA chain extension at $72{ }^{\circ} \mathrm{C}$ for $2.5 \mathrm{~min}$. The amplified cDNA products were subsequently cloned into the pbluescript/ $\mathrm{KS}+(\mathrm{pBS})$ vector (Stratagene, La Jolla, USA) to generate the plasmid constructs pBS/Ad (NS3), and pBS/Ad (NS3 $\Delta 50)$ (Tab. I). Constructs were sequenced to confirm the BVDV-specific nature of the amplified product.

\subsection{Construction of AdV}

The procedures used were carried out essentially as described [15]. First, the cDNA NS3 and NS3 $\Delta 50$-encoding sequences were excised from plasmids pBS/Ad (NS3), and $\mathrm{pBS} / \mathrm{Ad}(\mathrm{NS} 3 \Delta 50)$, respectively, with restriction enzyme PmeI, purified, and subcloned in the adenovirus transfer vector AdTR5-DC-GFPq digested with EcoRV. This transfer vector enables the gene of interest to be expressed from a tetracycline-inducible promoter in di-cistronic configuration coexpressing GFP and the BVDV protein of interest. Thereafter, 293A cells were cotransfected with FseI-restricted transfer vector, and the $C l a \mathrm{I}$-restricted $\mathrm{Ad} 5 / \Delta \mathrm{E} 1 \Delta \mathrm{E} 3$ viral DNA to generate recombinant viruses by in vivo homologous recombination between overlapping sequences of linearized transfer vectors pAdTR5-DC-GFPq, and $\mathrm{Ad} 5 / \Delta \mathrm{E} 1 \Delta \mathrm{E} 3$ viral DNA $[25,26]$. Adenoviral plaques were screened 10 to 15 days after cell transfection by monitoring basal GFP expression by fluorescence microscopy. AdV were then purified by three further rounds of plaque isolation on BMAdE1 cells, and expanded as described [26]. Titers of the AdV stocks were estimated by a method based on the measurement of the GFP signal by cytofluorometry [25]. AdV stocks with gene transfer unit (GTU) titers ranging from $1-3 \times 10^{10}$ per $\mathrm{mL}$ were obtained after 20 -fold virus concentration.

\subsection{Expression of BVDV NS3 and NS3 $\triangle 50$ in mammalian cells by Western immunoblotting}

The BVDV NS3 antiserum used in this study was raised in rabbits against recombinant NS3 $\Delta 50$ (rNS3 $\Delta 50$ ) fusion proteins expressed in Escherichia coli [16, 20] using the pEt-21b expression vector (Novagen, Madison, USA). The cells were washed in phosphate-buffered saline (PBS) solution, $\mathrm{pH} 7.3$, and lysed in standard SDS-PAGE sample buffer. Total cell extract proteins were fractionated by $15 \%$ SDS-PAGE under reducing conditions and electrotransferred onto nitrocellulose membranes. Western immunoblotting on cell extracts was performed using, as the blocking reagent solution, $5 \%$ nonfat dried milk solids and $0.05 \%$ Tween 20 in PBS [16]. The blot was incubated with rabbit preimmune serum and BVDV NS3 $\Delta 50$-specific antiserum for $2 \mathrm{~h}$ at room temperature. The membranes were then washed three times in PBS before adding a peroxidase-conjugated goat anti-rabbit immunoglobulin $\mathrm{G}$ (whole molecule) for $1 \mathrm{~h}$ at room temperature. The immunological reactivity was revealed by enhanced chemiluminescence (ECL; Perkin Elmer, Boston, USA). Thereafter, the membranes were stripped off for actin immunostaining (Chemicon, Temecula, USA).

\subsection{Apoptosis analysis}

\subsubsection{AdV infection}

In order to determine the apoptotic capability of BVDV NS3 or NS3 $\triangle 50$, one-day old (sub-confluent) A549tTA cells were infected with each of the AdV expressing the respective BVDV protein at a multiplicity of infection (MOI) value of 100 200 GTU/cell [25]. The most appropriate infective dose with no or minimal background toxic effect on the cell culture monolayer was determined for each virus stock. An AdV only expressing GFP (AdV-GFP) with the same genetic background as the AdV expressing the BVDV protein was 
used as a negative control, whereas cells treated with actinomycin D $(50 \mu \mathrm{g} / \mathrm{mL})$ were used as a positive control of apoptosis [3]. Culture medium used for mock infection served as an additional negative control. The cells were analyzed for BVDV gene expression or cell apoptosis indicators (see below) at different time periods post infection (pi).

For Vero, Cf2Th, and MDBK cell infection, the cells were first exposed to an AdV expressing the tetracycline-regulated transactivator (AdV-tTA) [25] $3 \mathrm{~h}$ prior to adding the $\mathrm{AdV}$ expressing the BVDV NS3 350 or GFP alone. The cells were then analyzed for apoptosis by flow cytometry as described below.

\subsubsection{Flow cytometry}

Single cell suspensions (including adherent and nonadherent cells) were prepared at various time points following AdV infection by trypsinization, centrifugation and resuspending in $200 \mu \mathrm{L}$ of a PBS solution containing $25 \mu \mathrm{g} / \mathrm{mL}$ propidium iodide (PI), $0.06 \%$ saponin, $2.5 \mathrm{U} / \mathrm{mL}$ RNAse A, and $20 \mu \mathrm{M}$ EDTA for $20 \mathrm{~min}$ before cytometry analysis using a fluorescence-activated cell sorter (FACScan, Becton Dickinson, Mississauga, Canada). Cell debris were excluded from the analyses by the conventional scatter gating method. The cells with nuclei doublets were also excluded from the analyses using pulse processor boards. Ten thousand events per sample were analyzed using the Cell Quest software system (Becton Dickinson).

\subsubsection{DNA fragmentation}

The fragmentation of cellular DNA was analyzed by visualizing oligonucleosomalsized DNA fragments (DNA ladder formation) [3]. In situ DNA fragmentation from cells that were grown in coverslips (8-well Labtek chamber) was assessed using a colorimetric Tdt-mediated dUTP nick end labeling (TUNEL) commercial kit (In situ Cell Death Detection, POD; Roche Diag- nostics, Mannheim, Germany), according to the supplier's instructions.

\subsubsection{Detection of poly(ADP-ribose) polymerase (PARP) cleavage}

Adherent cells collected at various time points following treatment (actinomycin D or cell infection with each AdV) were washed with PBS, pooled with detached cells, lysed in standard SDS-PAGE sample buffer containing $6 \mathrm{M}$ urea, and sonicated for $15 \mathrm{~s}$ on ice. Cell proteins were fractionated by $8 \%$ SDS-PAGE and electrotransferred onto nitrocellulose membranes. Immunoblotting was performed as above by using, as the primary antibody, a PARP monoclonal antibody (C-2-10; BioVision Inc., Mountain View, USA), and, as the secondary antibody, a peroxidase-conjugated goat anti-mouse immunoglobulin $\mathrm{G}(\mathrm{H}+\mathrm{L}$ chains). The membranes were developed by ECL.

\subsubsection{Caspase enzymatic assays, and detection of pro-caspase cleavage}

Caspase- $3,-8$, and -9 colorimetric assays were performed according to the supplier's instructions (R \& D Systems, Minneapolis, USA). Briefly, A549tTA cells infected with the relevant AdV were lysed at $40 \mathrm{~h}$ pi in the caspase buffer ( $50 \mathrm{mM}$ Hepes, $150 \mathrm{mM}$ $\mathrm{NaCl}, 20 \mathrm{mMEDTA}, 1 \mathrm{mMDTT}$ ) for $15 \mathrm{~min}$ on ice. The protein concentrations were determined using a commercial kit (Bio-Rad, Mississauga, Canada). The assays were performed three times in duplicates $(100 \mu \mathrm{g}$ protein per $50 \mu \mathrm{L}$ of cell lysis buffer) in microplates using the appropriate caspase-3 (Z-DEVDpNA), -8 (Z-IETD-pNA), and -9 (Z-LEHDpNA) colorimetric substrates. The samples were analyzed in an ELISA microplate reader (Bio-Rad) at $405 \mathrm{~nm}$. An increase of the $\mathrm{OD}_{405 \mathrm{~nm}}$ of $>100 \%$, when compared to that of the mock-infected cell control, was considered as being positive.

For detection of pro-caspase cleavage, the cells were infected with AdV-NS3 and 
collected as above at 24 and $48 \mathrm{~h}$ pi. The cells were then lysed in lysis buffer $(50 \mathrm{mM}$ HEPES, pH 7.5, $150 \mathrm{mM} \mathrm{NaCl}, 20 \mathrm{mM}$ EDTA, $0.2 \%$ TritonX-100, $50 \mathrm{mM}$ DTT) supplemented with a cocktail of protease inhibitors (Roche Diagnostics). Proteins $(50 \mu \mathrm{g})$ were fractionated by $15 \%$ SDSPAGE and immunoblotted as above. Caspase-3 and -9 specific monoclonal antibodies were purchased from R \& D Systems, and caspase- 8 specific polyclonal antibodies were purchased from BD Biosciences (Mississauga, Canada).

\subsubsection{Caspase-specific peptide inhibitors, and the R1 subunit of herpes simplex virus type 2 ribonucleotide reductase (HSV2-R1) and hsp70 biological inhibitors}

A549tTA cells were plated at a density of $2.5 \times 10^{4}$ cells per well into each well of a 24-well plate. The adhered cells (3-4 h of adsorption) were treated with caspase inhibitors $(50 \mu \mathrm{M}) \mathrm{Z}$-VAD-FMK (pancaspase), Z-IETD-FMK (caspase-8-specifc inhibitor), or Z-LEHD-FMK (caspase-9specific inhibitor) (R \& D Systems), and, two hours later, infected with the BVDV NS3 $\Delta 50$-expressing AdV. The cells were replenished with the caspase inhibitors $24 \mathrm{~h}$ later, and collected at $48 \mathrm{~h}$ after infection with AdV for cytometry analysis. For HSV2R1 and hsp70 inhibition assays, the cells were infected with both the BVDV AdV and an AdV expressing either HSV2-R1 (AdTR5-R1; AdV-HSV2-R1) [24] or hsp70 (AdTR5-hsp70-DC-BFP; AdV-hsp70) [26], and then tested at $48 \mathrm{~h}$ pi for apoptosis by cytometry analysis.

\subsubsection{Analysis of cytochrome c translocation}

For cytochrome $\mathrm{c}$ analysis, mock-infected and AdV-NS3 $\Delta 50$-infected A549tTA cells were collected at various times pi. As a control of cytochrome $\mathrm{c}$ release, the cells were exposed to staurosporine $(100 \mu \mathrm{g} / \mathrm{mL})$ [33].
Subcellular fractionation of the proteins was performed according to previously described protocols $[8,10]$ with slight modifications. Briefly, the cells were trypsinized, centrifuged $(800 \times g$ for $5 \mathrm{~min})$, lysed in a digitonin buffer $(1 \mathrm{mg} / \mathrm{mL}$ digitonin, $20 \mathrm{mM}$ Hepes, pH 7.4, $250 \mathrm{mM}$ sucrose) supplemented with protease inhibitors for $1 \mathrm{~min}$, and microcentrifuged at $11000 \times g$ for 2 min. The supernatant (cytosolic fraction) was collected, whereas the cell pellet was resuspended in mitochondria buffer $(0.2 \%$ TritonX-100, $150 \mathrm{mM} \mathrm{NaCl}, 30 \mathrm{mM}$ Tris$\mathrm{HCl} \mathrm{pH}$ 7.2) supplemented with protease inhibitors, and incubated for $10 \mathrm{~min}$ at $4{ }^{\circ} \mathrm{C}$. The cell mixture was then microcentrifuged for $15 \mathrm{~min}$ at $11000 \times g$, and the supernatant containing the mitochondria fraction was collected. Proteins $(50 \mu \mathrm{g})$ were fractionated by $15 \%$ SDS-PAGE and immunoblotted as above using, as the primary antibody, an anti-human cytochrome c mouse monoclonal antibody ( $\mathrm{R} \& \mathrm{D}$ Systems), and, as the secondary antibody, a peroxidase-conjugated goat anti-mouse immunoglobulin $\mathrm{G}(\mathrm{H}+\mathrm{L}$ chains). The membranes were developed by ECL as above. Thereafter, the membranes were stripped off for actin immunostaining.

\section{RESULTS}

\subsection{Cytopathogenicity correlates with BVDV NS3 and NS3 $\triangle 50$ expressed from AdV}

Following infection of A549tTA cells with each of the BVDV protein-expressing AdV and the control AdV-GFP, the GFP signal was generally visible under fluorescence microscopy around 6 to $8 \mathrm{~h}$ pi. Along with the expression of GFP, the cells infected with each AdV carrying the BVDV sequences showed the first evidence of morphological changes (cell rounding and shrinking in size and cell detachment in cell culture supernatant) from 18 to $24 \mathrm{~h}$ pi. Microscopic observations at $40 \mathrm{~h}$ pi showed that most of the shrinking cells carried the characteristic apoptosis nucleus chromatin 
A

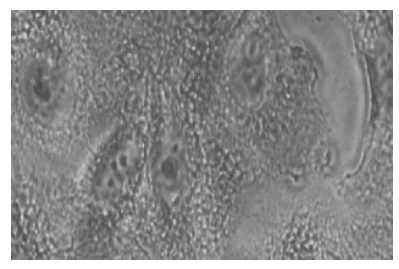

AdV-GFP

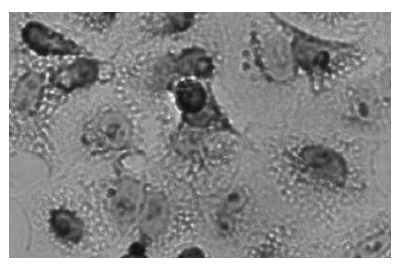

AdV-NS3

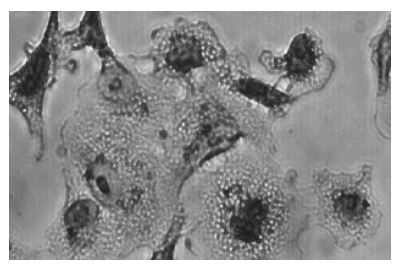

AdV-NS3 $\triangle 50$

B

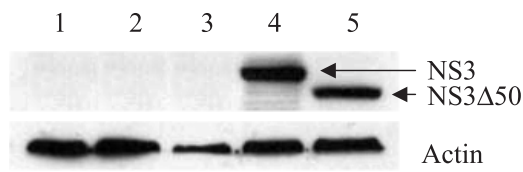

Figure 1. Morphological changes in cells upon expression of BVDV NS3 and NS $3 \Delta 50$. (A) Changes in cell morphology (400x enlargement) induced by BVDV NS3 and NS3 $\triangle 50$ proteins. A549tTA cells were infected with GFP-expressing AdV (AdVGFP), BVDV NS3-expressing AdV (AdVNS3) or BVDV NS3 $\Delta 50$-expressing AdV (AdV-NS3 450 ), and photographed after $40 \mathrm{~h}$ of incubation. In situ cell DNA fragmentation was assessed using a colorimetric Tdtmediated dUTP nick end labeling (TUNEL) commercial kit. (B) Expression analysis of BVDV NS3 and NS3 $\Delta 50$ proteins in A549tTA cells by Western immunoblotting. The cells were mock-infected $(40 \mathrm{~h}$ of incubation), treated with actinomycin D ( $24 \mathrm{~h}$ of incubation), or infected with the relevant AdV (40 h of incubation). Lane 1, mockinfected cells; lane 2, cells infected with AdVGFP; lane 3, actinomycin D treated-cells; lane 4, cells infected with AdV-NS3; lane 5, cells infected with AdV-NS3 $\Delta 50$. As a control for protein concentration, the membranes were stripped off for actin immunostaining. condensation with a reduction in cell volume (Fig. 1A). No significant CPE was observed in mock-infected cells (not shown) and in cells infected with AdV-GFP (Fig. 1A). In contrast, CPE was readily observed in cells treated with actinomycin D, which was used as a positive control of apoptosis.

Along with the appearance of CPE, both BVDV NS3 and NS3 450 proteins, as determined by Western immunoblotting, were expressed from cells infected with each of the AdV (Fig. 1B, lanes 4 and 5, respectively) at relatively similar levels. No immune reactivity was obtained when the cell proteins were exposed to the rabbit pre-immune serum. Expression of BVDV NS3 $\triangle 50$ and NS3 proteins, as determined by confocal fluorescence microscopy, was observed in cells that concomitantly expressed GFP (not shown), thereby demonstrating the effectiveness of the dicistronic adenovirus expression system used in this study. Therefore, it was concluded that expression of BVDV NS3 or NS3 $\Delta 50$ from each AdV correlated with CPE in infected cells. 
A
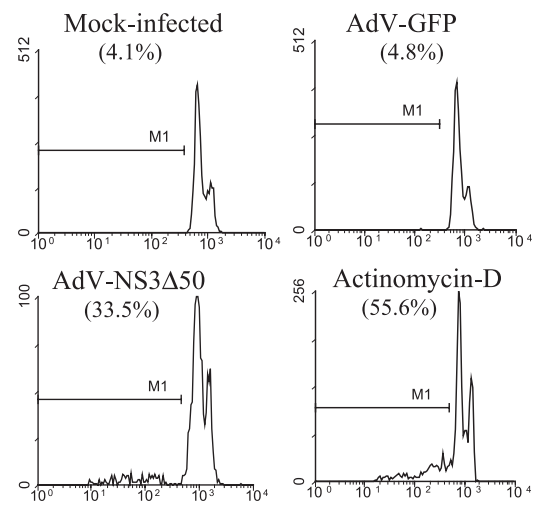

B

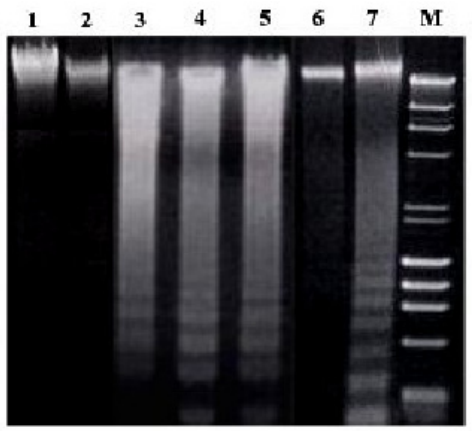

C

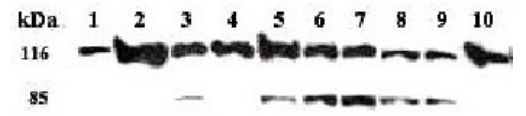

Figure 2. Apoptosis assessment in cells expressing the BVDV-NS3 or NS3 350 proteins. (A) Determination of subdiploid DNA content of apoptotic A549tTA cells by flow cytometry. Representative histograms of cell DNA fragmentation obtained after $40 \mathrm{~h}$ of cell incubation are shown. A549tTA cells were mock-infected, treated with actinomycin D, or infected with GFPexpressing AdV (AdV-GFP) or BVDV NS3 $\Delta 50$ expressing AdV (AdV-NS3 450$)$. Single cell suspension were permeabilized/stained with a saponin/propidium iodide (PI)-containing solution before flow cytometry analysis. PI fluorescence was detected at $660 \mathrm{~nm}$ ( $\times$ axis). M1 refers to areas showing lower DNA content. The percentages of apoptotic cells are shown. (B) Cell DNA oligonucleosomal fragmentation analysis as determined on ethidium bromide stained agarose gel. Lane 1, mock-infected A549tTA cells ( $40 \mathrm{~h}$ of incubation); lane 2, cells infected with AdV-GFP (40 h of incubation); lane 3, actinomycin D-treated cells (30 h of incubation); lane 4, cells infected with AdV-NS3 (40 h of incubation); lane 5, cells infected with AdV-NS3 $\triangle 50$ (40 h of incubation); lane 6, mock-infected MDBK cells (72 $\mathrm{h}$ of incubation); lane 7, MDBK cells infected with BVDV NADL strain (72 $\mathrm{h}$ post-infection). M: HaeIII-digested $\phi X-174$ and HindIII- $\lambda$ replicativeform DNA as molecular mass markers. (C) Kinetics of the expression of the PARP cleavage product by Western immunoblotting analysis. Lanes 1 and 2 refer to mock-infected A549tTA cells after 12 and $60 \mathrm{~h}$ of incubation, respectively; lane 3 refers to actinomycin D-treated cells after $40 \mathrm{~h}$ of incubation; lanes 4 to 9 refer to cells infected with AdV-NS3 $\Delta 50$ at $12,24,30,40,48$, and $60 \mathrm{~h}$ postinfection, respectively; lane 10 refers to cells infected with AdV-GPF at $40 \mathrm{~h}$ post-infection.

or treated with actinomycin D (used as an apoptosis positive control) (Fig. 2A). In contrast, no sub G0/G1 peak was observed in cells mock-infected or infected with AdVGFP, thereby indicating the absence of apoptosis in these cells. Similar results of BVDV NS3 $\Delta 50$-induced apoptosis were obtained in Vero, Cf2Th and MDBK indicator cells (not shown).

Since the oligonucleosomal DNA ladder of multiples of 180-200 base pairs in apoptotic cells is considered a hallmark of apoptosis, we carried out DNA fragmentation assays. Figure 2B shows typical DNA fragmentation in cells infected with each of the apoptosis from cells infected with the NS3 (not shown) and NS3 $\Delta 50$-expressing AdV, 
AdV expressing either BVDV NS3 or NS3 $\Delta 50$ (lanes 4 and 5, respectively). DNA fragmentation was observed in cells treated with actinomycin D (lane 3) or in MDBK cells infected with the NADL strain of BVDV (lane 7) that was used as an additional positive control of apoptosis. In contrast, no DNA fragmentation was observed in the mock-infected and AdV-GFP-infected negative control cell cultures (lanes 1 and 2 , respectively). To confirm, by an independent means, the cell DNA fragmentation, a colorimetric TUNEL assay was performed to detect in situ DNA fragmentation. Labeling of cell nuclei typical of DNA fragmentation was readily detected in cells infected with AdV expressing either BVDV NS3 or NS3 $\Delta 50$ (Fig. 1A). In contrast, no DNA labeling was detected in cells infected with the AdV-GFP negative control.

\subsection{Cleavage of the death substrate, PARP}

It is well known that chromosome DNA fragmentation requires the activation of cysteine proteases of the interleukin-1 $\beta$ converting enzyme (ICE), termed the caspases, leading to the cleavage of various death substrates, including the $116 \mathrm{kDa}$ PARP. The kinetics of the expression of the PARP cleavage product $(85 \mathrm{kDa})$, as determined by Western immunoblotting, was then conducted from cells infected with BVDV NS3 $\Delta 50$-expressing AdV. As shown in Figure 2C, evidence of the PARP cleavage was observed from $24 \mathrm{~h}$ pi at the time when CPE was readily apparent, and continued to $60 \mathrm{~h}$ pi. PARP cleavage was observed in cells treated with actinomycin D (lane 3). PARP cleavage was also observed in cells infected with the BVDV NS3-expressing AdV (not shown). Altogether, the results clearly indicate that BVDV NS3 and NS3 $\triangle 50$ induced apoptosis is mediated through a caspase activation pathway. Finally, since the results also indicated that the first $\mathrm{NH} 2$ terminal fifty amino acids of NS3 are dispensable for its apoptotic capability, further

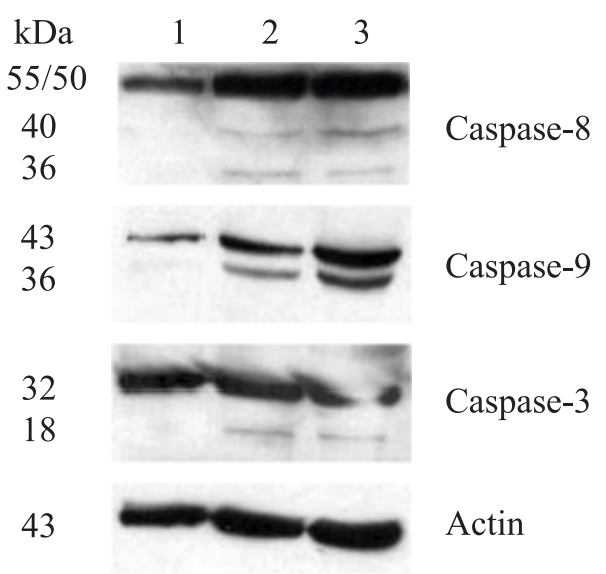

Figure 3. Detection of pro-caspase cleavage products by Western immunoblotting. A549tTA cells were mock-infected, or infected with BVDV NS3 $\Delta 50$-expressing AdV (AdV-NS3 $\Delta 50$ ). Lane 1, mock-infected cells after $48 \mathrm{~h}$ of incubation; lanes 2 and 3 refer to cells infected with AdVNS $3 \Delta 50$ and collected at 24 and $48 \mathrm{~h}$ post-infection, respectively. As a control for protein concentration, the membranes were stripped off for actin immunostaining. The molecular weights of the expected cleavage products are indicated in the left margin.

experiments were conducted with the BVDV NS3 $\Delta 50$-expressing AdV.

\subsection{Caspase activation and caspase inhibition by peptide inhibitors}

In light of the results indicating a caspase activation pathway for the NS3 and NS3 $\Delta 50$-induced apoptosis, we examined which caspase was activated in this apoptotic process. As determined in colorimetric caspase activation assays, an activation of caspase- 3 and -8 was detected increasing until it reached a maximum of $>300 \%$ (as compared with that of the mock- and AdVGFP-infected cell negative controls) at $48 \mathrm{~h}$ pi with the BVDV NS3 $\Delta 50$-expressing AdV. Interestingly, an increase of caspase- 9 activation, albeit to a lower level of 
Table II. Inhibition of BVDV NS3 $\Delta 50$-induced apoptosis by peptide or biological inhibitors.

\begin{tabular}{lccccc}
\hline $\begin{array}{l}\text { Recombinant } \\
\text { adenovirus } \\
\text { (AdV) }\end{array}$ & Z-VAD-FMK & Z-IETD-FMK & Z-LEHD-FMK & HSV2-R1 & hsp70 \\
\cline { 2 - 6 } & $70.39^{*} \pm 3.51$ & $64.40^{*} \pm 6.83$ & $9.74 \pm 5.45$ & $80.55^{*} \pm 7.50$ & $8.07 \pm 22.60$ \\
\hline AdV-FasL & $72.07^{*} \pm 1.41$ & $51.06^{*} \pm 4.94$ & $42.77^{*} \pm 9.14$ & $81.05^{*} \pm 4.00$ & $63.73^{*} \pm 3.72$ \\
\hline AdV-NS3 $\Delta 50$ & &
\end{tabular}

a Peptide inhibitors: Z-VAD-FMK, pan-caspase inhibitor; Z-IETD-FMK, caspase-8-specific inhibitor; Z-LEHD-FMK, caspase-9-specific inhibitor. Biological inhibitors: HSV2-R1, inhibitor of apoptosis associated with caspase-8 activation; hsp70, inhibitor of apoptosis associated with caspase-9 activation.

$\mathrm{b}$ The results are means (percentages of inhibition when compared to control cells with no inhibitor) \pm SD for three independent experiments (duplicate samples for each experiment). The significant values different from the control cells with no inhibitors are indicated by an asterisk $(p<0.001)$.

200\%, was also observed. Caspase- 3 and -8 activities, but not caspase- 9 activity, were measurable in cells infected with an AdV expressing FasL (AdV-FasL) that was used as a positive control of caspase- 8 activation. Because caspase substrates generally lack enzymatic specificity, we wished to verify whether the selected pro-caspases $-8,-9$, and -3 could be subjected to enzymatic cleavage, an indication of caspase activation. As shown in Figure 3, cleavage of all pro-caspases tested $(-8,-9$, and -3$)$ was detectable by $24 \mathrm{~h}$ pi and readily at the experiment endpoint of $48 \mathrm{~h}$ pi.

To demonstrate the importance of caspase activation in apoptosis induction, experiments were conducted with caspase-specific peptide inhibitors. As shown in Table II, 72 and $51 \%$ of inhibition of apoptosis were obtained in BVDV NS3 350 -expressing AdV-infected cells treated with the pan-caspase (Z-VADFMK), and caspase-8-specific (Z-IETDFMK) inhibitors, respectively. Lower but significant $(p<0.001)$ inhibition of $42.7 \%$ was also observed in cells treated with caspase-9-specific inhibitor (Z-LEHD-FMK). By comparison, 70, 64 and $9.7 \%$ of inhibition of apoptosis were obtained with the pan-caspase, caspase-8-specific and caspase-9-specific inhibitors, respectively, from cells infected with the AdV-FasL that were used as a control of caspase-8 activation. Thus, the results obtained here suggested that the BVDV NS3 $\Delta 50$ induces apoptosis that correlated with both caspase- 8 and caspase- 9 activation.

\subsection{Apoptosis inhibition by HSV2-R1 and hsp70}

To confirm by independent and more specific means the results obtained with the caspase peptide inhibitors, we determined the capability of known anti-apoptotic proteins to inhibit the NS3 $\Delta 50$-induced apoptotic process. Here, we selected HSV2-R1, which specifically blocks caspase-8-mediated apoptosis, and hsp70, which specifically blocks the cytochrome c release-dependent caspase-9 activation pathway. As shown in Table II, HSV2-R1, when expressed in cells infected with AdV-NS3 $\Delta 50$, inhibited by $81 \%$ the NS3 $\Delta 50$-induced apoptosis, as determined by cytometry analysis. This inhibition level was comparable to the one obtained in cells co-infected with AdVHSV2-R1 and AdV-FasL that were used as a control for the inhibition of caspase- 8 activation. On the contrary, hsp70 inhibited by $63.7 \%$ the NS3 $\Delta 50$-induced apoptosis. As an additional negative control, no inhibition of apoptosis was observed in cells co-infected with AdV-FasL and AdVhsp70. Thus, we concluded that caspase- 8 and caspase- 9 activation are with no doubt associated with BVDV NS3 $\Delta 50$ - and presumably NS3-induced apoptosis. 


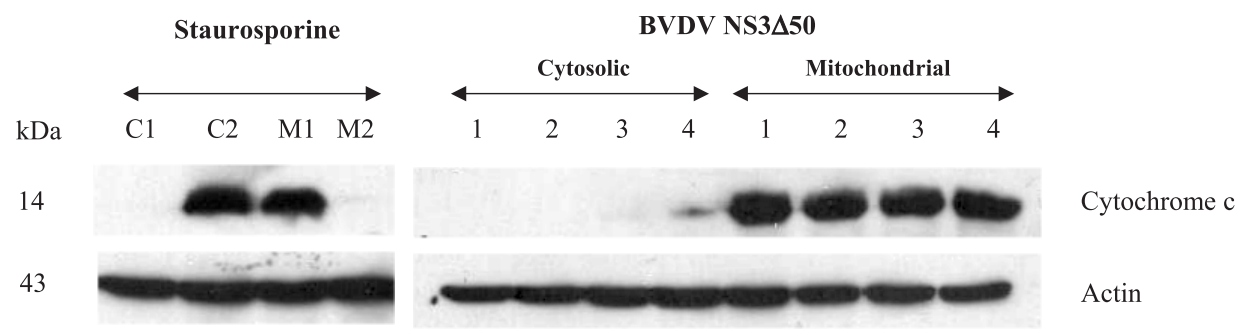

Figure 4. Localization of cytochrome $\mathrm{c}$ in subcellular fractions of mock-infected cells, A549tTA cells infected with BVDV NS3 $\Delta 50$-expressing AdV (AdV-NS3 $\Delta 50$ ) or cells exposed to staurosporine (positive control of cytochrome $\mathrm{c}$ translocation) at different time points by Western immunoblotting. As a control for protein concentration, the membranes were stripped off for actin immunostaining. Staurosporine: Lanes C1 and C2, and M1 and M2 refer to the cytosolic and mitochondrial fractions of the cells exposed for 1 or $4 \mathrm{~h}$, respectively. BVDV NS3 $\Delta 50$ : Lane 1, mock-infected cells after $48 \mathrm{~h}$ of incubation; lanes 2, 3, and 4 refer to cells infected with AdV-NS3 $\Delta 50$ at 16, 24, and $48 \mathrm{~h}$ post-infection, respectively. The molecular weights of the expected products are indicated in the left margin.

\subsection{Detection of cytochrome c translocation}

In light of the results obtained above, we wished to determine whether the release of cytochrome c in the cytosol, a step generally involved in caspase- 9 activation, could have occurred in cells infected with AdV-NS3 450 . Time course studies showed that cytochrome $\mathrm{c}$ was released from the mitochondria in the cytosol by $24 \mathrm{~h}$ pi (as shown by the faint band) and readily at the experiment end-point of $48 \mathrm{~h}$ pi (Fig. 4). As a control of the caspase-9 mitochondriadependent activation pathway, the release of cytochrome $\mathrm{c}$ in the cytosol was readily observed from cells treated with staurosporine from $1 \mathrm{~h}$ post-exposition.

\section{DISCUSSION}

Caspases, present in mammalian cells as inactive protease precursors (the so-called pro-caspases), are grouped into upstream initiator caspases (caspases-8 and -9) and in downstream effector caspases (such as caspase- $3,-6$, or -7). Initiator caspases- 8 and -9 are first activated in response to apoptotic stimuli and are responsible for processing and activation of effector caspases which, in turn, mediate apoptosis by cleaving cellular substrates that are indispensable for cell survival [32, 41, 45]. In this study, we used an AdV-inducible expression system that allowed us to express both the NS3 and the NS3 350 of the pestivirus BVDV. The system was also used to directly determine the involvement of these proteins in the induction of apoptosis in vitro. The results have shown that the expression of both proteins (NS3 and NS3 $\triangle 50$ ) in cells infected with the respective $\mathrm{AdV}$ was clearly associated with the induction of CPE, cell DNA fragmentation typical of apoptosis, cleavage of the cellular substrate PARP, and/or activation of caspases.

Infection of cells in vitro with cp BVDV (whole virus) induces apoptosis through the caspase activation pathway $[14,46]$. In addition, oxydative stress [40], intracellular viral RNA accumulation [44], caspase-12associated ER stress [18], and mitochondriadependent caspase-9 activation [11] have been reported to be associated with $\mathrm{cp}$ BVDV-induced apoptosis. Here we show that NS3/NS3 $\triangle 50$ of BVDV induced apoptosis, and correlated with both caspase- 8 and caspase- 9 activation. Caspase- 8 and -9 activation was demonstrated by the measurement of caspase enzymatic activity 
and pro-caspase cleavage, and the inhibition results obtained with caspase-8 (Z-IETDFMK)- and caspase-9 (Z-LEHD-FMK)specific peptide inhibitors, and with biological HSV2-R1, and hsp70 inhibitors. HSV2-R1 blocks the activation of caspase- 8 by an unknown mechanism [24], whereas hsp70 blocks the caspase-9-associated apoptotic process by inhibiting the release of cytochrome $\mathrm{c}$ from mitochondria, or by a yetto-be-characterized mechanism that occurs downstream of caspase- 9 activation $[12,21$, 30]. The facts that both caspase- 8 and -9 were activated upon NS3 $\Delta 50$ expression following $\mathrm{AdV}$ infection, and that the BVDV NS3 350 -induced apoptosis correlated with cytochrome c release, suggest that caspase-8 likely acts indirectly at the mitochondria level, resulting in the release of cytochrome $\mathrm{c}$ from the mitochondria, and downstream activation of caspase- 9 and effector caspases, rather than directly activating caspase-3 [38]. Although other experiments are needed to unequivocally confirm this point, this interpretation is supported by the fact that inhibition of NS3 $\Delta 50$-associated caspase- 8 activation by HSV2-R1 resulted in inhibition of caspase-9 activation as well (as determined by the absence of the cleavage form of pro-caspase-9) (not shown). In any case, our results agreed with those reported by Grummer et al. [11] where caspase- 9 activation correlated with cytochrome c translocation to the cytosol in the induction of apoptosis with cp BVDV.

The caspase- 8 activation observed here in BVDV NS3-induced apoptosis was consistent with that reported by other authors [35] where the Langat virus NS3-induced apoptosis was also associated with caspase- 8 activation. The Langat virus is a flavivirus that, as BVDV, belongs to the Flaviviridae family. Langat NS3-associated caspase- 8 activation correlated with the binding of NS3 to caspase-8. Whether the BVDV NS3 protein indeed binds caspase8 has yet to be determined. This is an important issue to address because the Langat virus NS3 amino acid sequence only shows
$22 \%$ of identity (using the BioEdit software, 5.0.9 version, and the Blosum62 matrix) with that of the BVDV NS3. The same authors [35] also showed that the Langat virus NS3 protease domain located between amino acids 1 and 181 is involved in apoptosis. Whether the BVDV NS3 protease domain (located from amino acids 56 to 181, according to the Print Database accession number PR000729) is responsible for the apoptosis observed here has yet to be confirmed. Whatever the mechanisms would be, it is possible that the overall picture of the pestivirus NS3-induced apoptosis might be different from the Langat NS3induced apoptosis which, as opposed with the pestivirus NS3, appeared to be independent of caspase-9 activation [35].

In recent years, several authors have reported concomitant caspase- 8 and caspase-9 activation in various apoptotic systems with or without the release of cytochrome $\mathrm{c}$ from mitochondria [6, 17], further demonstrating the complex nature of the apoptotic process. Although the NS3/ NS3 $\Delta 50$ protein induces caspase-8- and -9dependent apoptosis, several lines of evidence suggest that several factors (cellular and/or viral) or activation pathways of apoptosis are likely to be involved in the BVDV-associated apoptosis process. The facts that oxidative stress is observed in cells infected with cp BVDV [40], and that caspase-12-associated ER stress, a feature that was not addressed in this study, is observed in cp BVDV-induced apoptosis are consistent with the latter statement [18]. Caspase-12, which resides within the cytoplasmic side of the ER, is believed to play a central role in the ER stress-mediated apoptosis [31] and can activate caspase- 8 [7] which in turn stimulates, through Bid processing, cytochrome c release and activates caspase-9 [17]. Given its association with the membranes of ER [47], NS3 might also trigger apoptosis through caspase- 12 activation. On the contrary, monocytes/macrophages infected in vitro with cp BVDV released an interferon-like molecule and another yet to identify factor in the supernatant 
capable of priming uninfected macrophages for activation-induced apoptosis in response to lipopolysaccharide [2, 19, 34]. In addition, the glycoprotein $\mathrm{E}^{\mathrm{rns}}$ of the pestivirus CSFV, that is also present in BVDV, has been reported to induce apoptosis in vitro [9]. Whether the $\mathrm{E}^{\mathrm{ms}}$ of BVDV is associated with apoptosis remains to be investigated. Finally, it has been recently reported that ncp BVDV appears to inhibit apoptosis associated to cp BVDV [5]. Altogether, these reports argue for the involvement of several cellular/viral factors and/or pathways involved in the regulation of the BVDV apoptotic process at least in vitro.

The results presented here show that the BVDV NS3, and thereof BVDV NS3 $\Delta 50$, when expressed alone from an AdVinducible expression system, induces cell apoptosis in vitro. However, the exact role of NS3 in the cytopathogenicity of BVDV in vitro and in vivo needs further clarification, particularly since the protein, in this study, was expressed without the presence of other BVDV proteins. Nonetheless, the BVDV NS3 is the first described pestivirus nonstructural protein per se able to induce apoptosis in vitro. Our results also showed that the first NH2-terminal fifty amino acids of NS3 are dispensable for the apoptotic capability of the protein, and that the NS3/ NS3 $\Delta 50$-induced apoptosis correlates with both caspase- 8 and caspase- 9 activation. Experiments are planned to further delineate the mechanisms of BVDV NS3-induced apoptosis and to determine which domains of NS3 are involved in the induction of apoptosis.

\section{ACKOWLEDGEMENTS}

This work was supported by an operating grant (\# 4638) from the Conseil de Recherches en Pêcherie et en Agro-alimentaire du Québec (CORPAQ) to D. Archambault and B. Massie. We gratefully acknowledge Claire Guilbault, Catherine Simard and Denis Flipo for technical assistance. M.-C. St-Louis was supported by a graduate studentship from the Fonds de la Nature et des Technologies du Québec (FNATQ). D.
Archambault was supported by a senior research scholarship from the Fonds de la Recherche en Santé du Québec (FRSQ). This is an NRC publication No. 37734.

\section{REFERENCES}

[1] Abed Y., St-Laurent G., Zhang H., Jacobs R.M., Archambault D., Development of a Western blot assay for detection of bovine immunodeficiency-like virus using capsid and transmembrane envelope proteins expressed from recombinant baculovirus, Clin. Diagn. Lab. Immunol. 6 (1999) 68-172.

[2] Adler B., Adler H., Pfister H., Jungi T.W., Peterhans E., Macrophages infected with cytopathic bovine viral diarrhoea virus release a factor(s) capable of priming uninfected macrophages for activation-induced apoptosis, J. Virol. 71 (1997) 3255-3258.

[3] Archambault D., St-Laurent G., Induction of apoptosis by equine arteritis virus infection, Virus Genes 20 (2000) 143-147.

[4] Archambault D., Béliveau C., Couture Y., Carman S., Clinical response and immunomodulation following experimental challenge of calves with type 2 noncytopathogenic bovine viral diarrhea virus, Vet. Res. 31 (2000) 215227.

[5] Bendfeldt S., Grummer B., Greiser-Wilke I. No caspase activation but overexpression of Bcl-2 in bovine cells infected with noncytopathic bovine virus diarrhoea virus, Vet. Microbiol. 96 (2003) 313-326.

[6] Bitzer M., Armeanu S., Prinz F., Ungerechts G., Wybranietz W., Spiegel M., Bernlohr C., Cecconi F., Gregor M., Neubert W.J., SchulzeOsthoff K., Lauer U.M., Caspase-8 and Apaf-1independent caspase- 9 activation in Sendai virus-infected cells, J. Biol. Chem. 277 (2002) 29817-29824.

[7] Breckenridge D.G., Nguyen M., Kuppig S., Reth M., Shore G.C., The procaspase- 8 isoform, procaspase-8L, recruited to the BAP31 complex at the endoplasmic reticulum, Proc Natl. Acad. Sci. USA 99 (2002) 4331-4336.

[8] Bronfman M., Loyola G., Koenig C.S., Isolation of intact organelles by differential centrifugation of digitonin-treated hepatocytes using a table Eppendorf centrifuge, Anal. Biochem. 255 (1998) 252-256.

[9] Bruschke C.J.M., Hulst M.M., Moormann R.J.M., van Run P.A., van Oirschot J.T., Glycoprotein $\mathrm{E}^{\mathrm{ms}}$ of pestiviruses induces apoptosis 
in lymphocytes of several species, J. Virol. 71 (1997) 6692-6696.

[10] Drapier J.C., Hirling H., Wietzerbin J., Kaldy P., Kuhn L.C., Biosynthesis of nitric oxide activates iron regulatory factor in macrophages, EMBO J. 12 (1993) 3643-3649.

[11] Grummer B., Bendfeldt S., Wagner B., Greiser-Wilke I., Induction of the intrinsic apoptotic pathway in cells infected with cytopathic bovine virus diarrhoea virus, Virus Res. 90 (2002) 143-153.

[12] Gurbuxani S., Schmitt E., Cande C., Parcellier A., Hammann A., Daugas E., Kouranti I., Spahr C., Pance A., Kroemer G., Garrido C., Heat shock protein 70 binding inhibits the nuclear import of apoptosis-inducing factor, Oncogene 22 (2003) 6669-6678.

[13] Hay S., Kannourakis G., A time to kill: viral manipulation of the cell death program, J. Gen. Virol. 83 (2002) 1547-1564.

[14] Hoff H.S., Donis R.O., Induction of apoptosis and cleavage of poly(ADP-ribose) polymerase by cytopathic bovine viral diarrhea virus infection, Virus Res. 49 (1997) 101-113.

[15] Jani A., Lochmuller H., Acsadi G., Simoneau M., Huard J., Garnier A., Karpati G., Massie B., Generation, validation, and large scale production of adenoviral recombinants with large size inserts such as $6.3 \mathrm{~kb}$ human distrophin cDNA, J. Virol. Methods 64 (1997) 111-124.

[16] Jeronimo C., Archambault D., Importance of $\mathrm{M}$-protein $\mathrm{C}$ terminus as substrate antigen for serodetection of equine arteritis virus infection, Clin. Diagn. Lab. Immunol. 9 (2002) 698703.

[17] Jimbo A., Fujita E., Kouroku Y., Ohnishi J., Inohara N., Kuida K., Sakamaki K., Yonehara S., Momoi T., ER stress induces caspase-8 activation, stimulating cytochrome c release and caspase- 9 activation, Exp. Cell Res. 283 (2003) 156-166.

[18] Jordan R., Wang L., Graczyk T.M., Block T.M., Romano P.R., Replication of a cytopathic strain of bovine viral diarrhea virus activates PERK and induces endoplasmic reticulum stress-mediated apoptosis of MDBK cells, J. Virol. 76 (2002) 9588-9599.

[19] Jungi T.W., Schweizer M., Perler L., Peterhans E., Supernatants of virus-infected macrophages prime uninfected macrophages for lipopolysaccharide-induced apoptosis by both interferon-dependent and independent mechanism, Pathobiology 67 (1999) 294-297.
[20] Kheyar A., Martin S., St-Laurent G., Timoney P.J., McCollum W.H., Archambault D., Expression cloning and humoral immune response to the nucleocapsid and membrane proteins of equine arteritis virus, Clin. Diagn. Lab. Immunol. 4 (1997) 648-652.

[21] Klein S.D., Brüne B., Heat-shock protein 70 attenuates nitric oxide-induced apoptosis in RAW macrophages by preventing cytochrome c release, Biochem J. 362 (2002) 635-641.

[22] Kummerer B.M., Tautz N., Becher P., Thiel H.-J., Meyers G., The genetic basis for cytopathogenicity of pestiviruses, Vet. Microbiol. 77 (2000) 117-128.

[23] Kyte J., Doolittle R.F., A simple method for dysplaying the hydropathic character of a protein, J. Mol. Biol. 5 (1982) 105-132.

[24] Langelier Y., Bergeron S., Chabaud S., Lippens J., Guilbault C., Sasseville A.M., Denis S., Mosser D.D., Massie B., The R1 subunit of herpes simplex virus ribonucleotide reductase protects cells against apoptosis at, or upstream of, caspase- 8 activation, $\mathrm{J}$. Gen. Virol. 83 (2002) 2779-2789.

[25] Massie B., Couture F., Lamoureux L., Mosser D.D., Guilbault C., Jolicoeur P., Bélanger F., Langelier Y., Inducible overexpression of a toxic protein by an adenovirus vector with a tetracycline-regulatable expression cassette, J. Virol. 72 (1998) 2289-2298.

[26] Massie B., Mosser D.D., Koutroumanis M., Vitté-Mony I., Lamoureux L., Couture F., Paquet L., Guilbault C., Dionne J., Chala D., Jolicoeur P., Langelier Y., New adenovirus vectors for protein production and gene transfer, Cytotechnology 28 (1998) 53-64.

[27] Mendez E., Ruggli N., Collett M.S., Rice C.M., Infectious bovine viral diarrhea virus (strain NADL) RNA from stable cDNA clones: a cellular insert determines NS3 production and viral cytopathogenicity, J. Virol. 72 (1998) 4737-4745.

[28] Meyers G., Thiel H.-J., Molecular characterization of pestiviruses, Adv. Virus Res. 47 (1996) 53-118.

[29] Mignotte B., Vayssiere J.L., Mitochondria and apoptosis, Eur. J. Biochem. 252 (1998) 1-15.

[30] Mosser D.D., Caron A.W., Bourget L., Meriin A.B., Sherman M.Y., Morimoto R.I., Massie B., The chaperone function of hsp70 is required for protection against stress-induced apoptosis, Mol. Cell. Biol. 20 (2000) 71467159.

[31] Nakagawa T., Zhu H., Morishima N., Li E., Xu J., Yankner B.A., Yuan J., Caspase-12 
mediates endoplasmic-reticulum-specific apoptosis and cytotoxicity by amyloid-beta, Nature 403 (2000) 98-103.

[32] Nicholson D.W., Caspase structure, proteolytic substrates, and function during apoptotic cell death, Cell Death Differ. 6 (1999) 10281042 .

[33] Pataer A., Chadas S., Hunt K.K., Roth, J.A., Swisher S.G., Adenoviral melanoma differentiation-associated gene 7 induces apoptosis in lung cancer cells through mitochondrial permeability transition-independent cytochrome c release, J. Thorac. Cardiovasc. Surg. 125 (2003) 1328-1335.

[34] Perler L., Schweizer M., Jungi T.W., Peterhans E., Bovine viral diarrhoea virus and bovine herpesvirus- 1 prime uninfected macrophages for lipopolysaccharide-triggered apoptosis by interferon-dependent and -independent pathways, J. Gen. Virol. 81 (2000) 881-887.

[35] Prikhod'ko G.G., Prikhod'ko E.A., Pletnev A.G., Cohen J.I., Langat flavirvirus protease NS3 binds caspase-8 and induces apoptosis, $\mathrm{J}$. Virol. 76 (2002) 5701-5710.

[36] Rao R.V., Hermel E., Castro-Obregon S., del Rio G., Ellerby L.M., Ellerby H.M., Bredesen D.E., Coupling endoplasmic reticulum stress to the cell death program. Mechanism of caspase activation, J. Biol. Chem. 276 (2001) 33869-33874.

[37] Rasper D.M., Vaillancourt J.P., Hadano S., Houtzager V.M., Seiden I., Keen S.L., Tawa P., Xanthoudakis S., Nasir J., Martindale D., Koop B.F., Peterson E.P., Thornberry N.A., Huang J., MacPherson D.P., Black S.C., Hornung F., Lenardo M.J., Hayden M.R., Roy S., Nicholson D.W., Cell death attenuation by "Usurpin", a mammalian DED-caspase homologue that precludes caspase-8 recruitment and activation by the CD-95 (Fas, APO-1) receptor complex, Cell Death Differ. 5 (1998) 271-288.

[38] Scaffidi C., Krammer P.H., Peter M.E., Isolation and analysis of components of CD95 (APO-1/Fas) death-inducing signaling complex, Methods 17 (1999) 287-291.
[39] Schulze-Osthoff K., Ferrari D., Los M., Wesselberg S., Peter M.E., Apoptosis signaling by death receptors, Eur. J. Biochem. 254 (1998) 439-459.

[40] Schweizer M., Peterhans E., Oxydative stress in cells infected with bovine viral diarrhoea virus: a crucial step in the induction of apoptosis, J. Gen. Virol. 80 (1999) 1147-1155.

[41] Shi Y., Mechanisms of caspase activation and inhibition during apoptosis, Mol. Cell 9 (2002) 459-470.

[42] St-Laurent G., Morin G., Archambault D. Detection of equine arteritis virus following amplification of structural and nontructural viral genes by reverse transcription-PCR, J. Clin. Microbiol. 32 (1994) 658-665.

[43] Thiel H.-J., Plagemann P.G.W., Moennig V., Pestiviruses, in: Fields B.N., Knipe D.M., Hewley P.M. (Eds.), Fields virology, Lippincott-Raven Publishers, 1996, pp. 1059-1073.

[44] Vassilev V.B., Donis R.O., Bovine viral diarrhea virus induced apoptosis correlates with increased intracellular viral RNA accumulation, Virus Res. 69 (2000) 95-107.

[45] Wolf B.B., Green D.R., Suicidal tendencies: apoptotic cell death by caspase family proteinases, J. Biol. Chem. 274 (1999) 2004920052.

[46] Zhang G., Aldridge S., Clarke M.C., McCauley J.W., Cell death induced by cytopathic bovine viral diarrhoea virus is mediated by apoptosis, J. Gen. Virol. 77 (1996) 1677-1681.

[47] Zhang G., Flick-Smith H., McCauley J.W., Differences in membrane association and subcellular distribution between NS2-3 and NS3 of bovine viral diarrhoea virus, Virus Res. 97 (2003) 89-102.

[48] Xu J., Mendez E., Caron P.R., Lin C., Murcko M.A., Collett M.S., Rice C.M., Bovine viral diarrhea virus NS3 serine proteinase: polyprotein cleavage sites, cofactor requirements, and molecular model of an enzyme essential for pestivirus replication, J. Virol. 71 (1997) 5312-5322. 